\title{
Overview and Performance of the Wavelength-shifting Optical Module (WOM)
}

\author{
The IceCube-Gen2 collaboration ${ }^{\dagger}$ \\ ${ }^{\dagger}$ http://icecube.wisc.edu/collaboration/authors/icrc17_gen2 \\ E-mail: peter.peiffer@uni-mainz.de
}

\begin{abstract}
The Wavelength-shifting Optical Module (WOM) is a novel photosensor concept developed in the context of the IceCube-Gen2 neutrino telescope, a next generation multi- $\mathrm{km}^{3}$ neutrino telescope at the South Pole. It provides a large photosensitive area with low detector noise and improved UV sensitivity. This is achieved by combining a wavelength-shifter (WLS) coated tube with two small, low-noise PMTs. Incident UV photons are absorbed by the WLS and re-emitted isotropically. A large fraction of the light is captured in the tube by total internal reflection and guided to the small PMTs. Through its cylindrical geometry, which is well matched to the drill holes, this concept results not only in an overall improved detection efficiency, but at the same time saves production and drilling cost. In this contribution we discuss the performance characteristics of the WOM and present the current status of the prototype development.
\end{abstract}

Corresponding autors: Peter Peiffer*1, Dustin Hebecker ${ }^{2}$

${ }^{1}$ University of Mainz, Germany,

${ }^{2}$ DESY Zeuthen, Germany

35th International Cosmic Ray Conference - ICRC 2017

12.-20. July 2017

Bexco, Busan, South-Korea

${ }^{*}$ Speaker. 


\section{Introduction}

IceCube [1] is currently the world's largest neutrino detector, with an instrumented volume of $1 \mathrm{~km}^{3}$. Installation was completed in 2010 and the detector has been running successfully in it's current configuration since 2011. IceCube-Gen2 is a planned extension to IceCube, both in highand low-energy detection capabilities. It encompasses an increased instrumented volume for high energy neutrinos [2] and an increased instrumentation density in the core (PINGU) [3] combined with improved photo-sensors. The Wavelength-shifting Optical Module (WOM) is one of the sensors under consideration for IceCube-Gen2. It aims especially at increasing the total number of detected photons by shifting UV photons into the optical range. This makes the module sensitive in the UV range, where the Cherenkov spectrum peaks in the ice. This results in an increased effective photosensitive area compared to the upgraded Digital Optical Module ( $\mathrm{pDOM}^{1}$ [4], another option for Gen2, see Fig. 1). At the same time, the WOM will have an order of magnitude lower noise than the currently used DOMs [1], which makes the WOM an ideal candidate for the low energy extension.



Figure 1: The wavelength-dependent absorptionand and effective scattering length for photons in the ice (dashed lines) and the Cherenkov-weighted effective area of the pDOM (red line) and the estimated, Cherenkov-weighted effective area of the WOM for 50\% detection efficiency (green line).

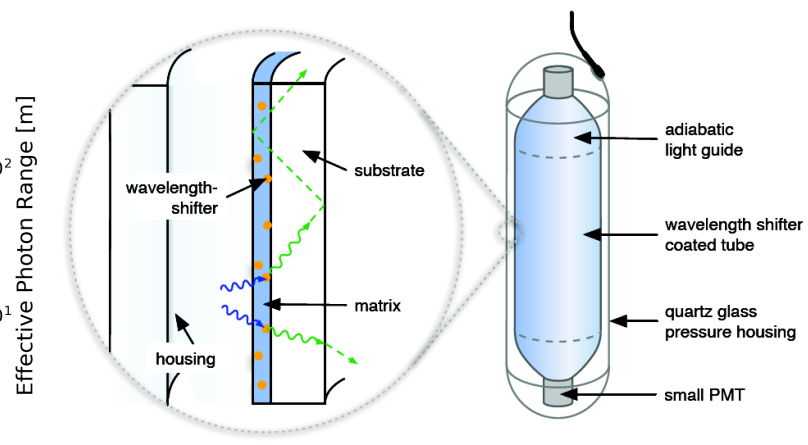

Figure 2: A schematic of the WOM: UV photons are absorbed in the WLS paint layer and are re-emitted as optical photons. If the incident angle of the emitted photons to the surface of the tube is smaller than the critical angle, they are trapped by total internal reflection and are guided along the tube to small, low-noise PMTs at the ends of the WOM tube.

The WOM, shown in Fig. 2, consists of a WLS-paint coated PMMA tube with an adiabatic light guide (ALG, [5]) at each end. These are connected to 3" PMTs and the entire assembly, including the read-out electronics, is housed in a quartz pressure vessel. UV photons are absorbed in the paint layer and are re-emitted as optical photons. This emission is isotropic and if the angle of the photon with respect to the tube surface is below the critical angle for total internal reflection, the photon is trapped and guided to the end of the tube: $\Theta_{c}=\arcsin \frac{n_{\text {air }}}{n_{P M A}} \approx 41.8^{\circ}$ for $n_{P M M A} \approx 1.5$. The theoretical maximum trapping efficiency is $74.5 \%$. At the end of the tube the light is concentrated by the ALG, which reduces the diameter of the tube while preserving the total cross section area. In theory, this allows a lossless concentration of the light. The paint is selected

\footnotetext{
${ }^{1}$ in this paper, DOM refers to the modules mounted in IceCube and pDOM to the upgraded version for Gen2
} 
for maximum overlap with the sensitivity of the PMT. The current prototype uses a Hamamatsu R12199-02 3" PMT, but other options are under investigation, including new super-bialkali PMTs. The advantages of this module are:

- Higher number of detected photons: The ice is transparent down to $\lambda=200 \mathrm{~nm}$ and the number of Cherenkov photons follows a $1 / \lambda^{2}$ distribution. The sensitive area is given by the tube surface $A_{0}$, which exceeds the sensitive area of the pDOM and can even be scaled up by lengthening the tube, at the cost of deteriorating timing resolution $(\approx 10 \mathrm{~ns}$ for a $1 \mathrm{~m}$ tube). The effective area (Fig.1) is given by $A_{\text {eff }}=A_{0} \cdot N_{p h}(\lambda) \cdot \varepsilon_{\text {capt }} \cdot \varepsilon_{\text {loss }}$ with $N_{p h}$ the integrated available spectrum, $\varepsilon_{\text {capt }}$ the capture efficiency and $\varepsilon_{\text {loss }}$ the loss factor due to attenuation.

- Lower noise: PMT noise scales with the photo-cathode area. The PMTs currently used in the WOM prototype have a dark noise of $\approx 30 \mathrm{~Hz}$ with a single-photoelectron-threshold at ice temperatures $\left(-10^{\circ} \mathrm{C}--40^{\circ} \mathrm{C}\right)$, compared to $\approx 500 \mathrm{~Hz}$ for the DOM-PMT.

- Lower energy threshold: the energy threshold of a Cherenkov detector is proportional to the effective sensitive area per unit volume and is limited by dark noise. Since the noise of the WOM is an order of magnitude lower and the effective sensitive area is larger than that of the (p)DOM, this module will allow the detection of lower energy events compared to IceCube with the same density of modules.

- Lower drilling cost: the cylindrical shape of the WOM matches the hole geometry better and has a much smaller diameter than the pDOM. This allows for smaller holes and saves on drilling time, energy and cost.

\section{Prototype development}

\subsection{Materials}

The WOM consists of the pressure vessel, the WLS coated tube plus light guides, the WLS paint and the PMTs. The pressure vessel has to be UV transparent and pressure resistant up to 700 bar. A commercial quartz tube with hemispherical end caps that fulfills these requirements has been selected. For the material of the WLS tube refractive index, compatibility with different WLS coatings and price were considered. In the end PMMA was chosen. The WLS needs to have a broad absorption spectrum in the UV range and minimal overlap between absorption and emission, so that it is transparent to its own emitted light. The WLS optimization has been described in [5]. Fig. 3 shows the relative absorption and emission spectrum of the current WLS. However, research into other WLS paints is ongoing.

\subsection{Adiabatic light guide}

The development of the adiabatic light guide (ALG) has been reported in [5]. Different methods of attaching the ALG to the WOM tube have been investigated ranging from various glues to solvent welding. Fig.4 shows the result of using UV curing glue. By visual inspection, most of the light reaches the ends of the ALGs. However, the gluing lines are visible due to scattered light, signifying a small light loss there. Investigations are ongoing, whether the connection can be improved by solvent welding or a monolithic production of a WOM-tube with adiabatic ends. 


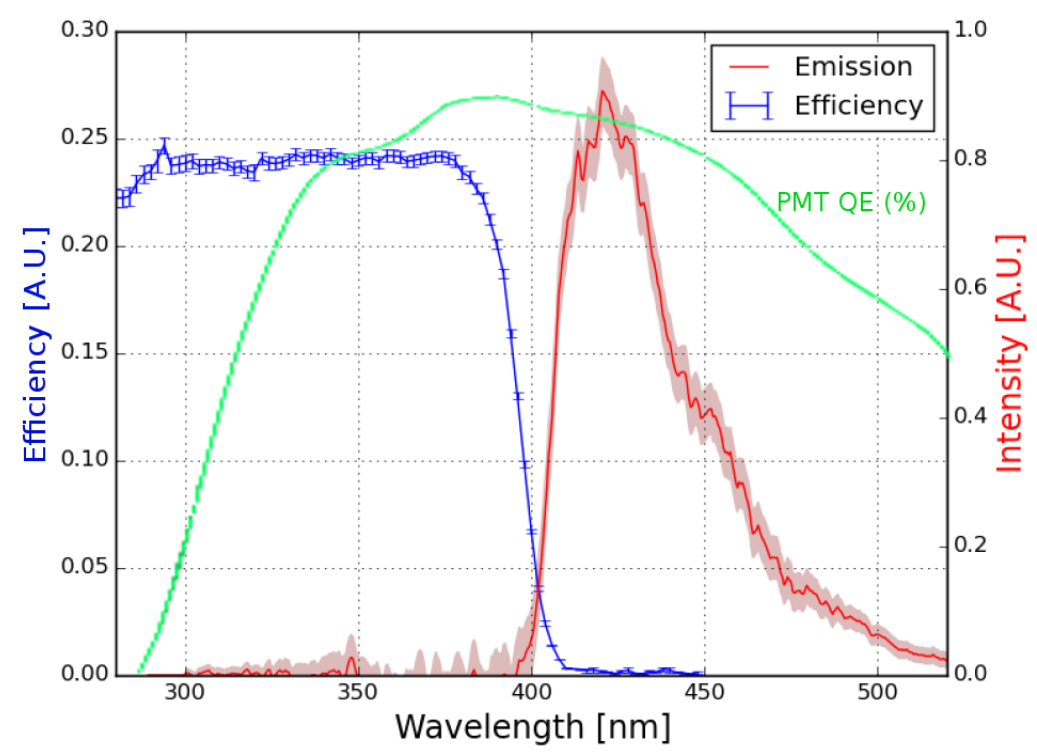

Figure 3: The relative shifting-efficiency and emission spectra of the WLS paint currently used in the WOM.

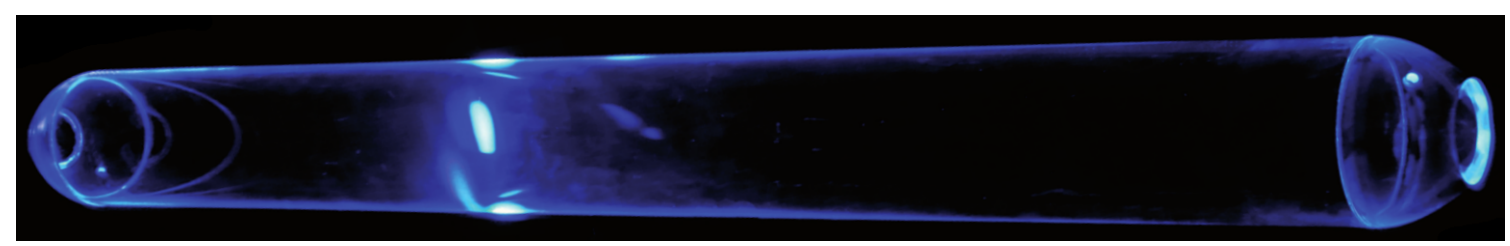

Figure 4: A WOM tube under UV light with two adiabatic light guides glued to it using UV-curing glue.

\subsection{Mounting}

To mount the WLS tube and the PMTs inside the pressure vessel, a holding structure has been developed. The end of the ALG is machined to match the curvature of the PMT, to which it is optically connected with a thin layer of optical gel. Around the ALG and the PMT is an aluminum jacket glued to the end of the WLS tube. Springs are attached to the jacket and to the base of the PMT, pulling the PMT against the ALG. In this way constant optical contact is ensured even during thermal contraction. The entire tube $+\mathrm{ALG}+\mathrm{PMT}$ construction is inserted into the pressure vessel, which is closed with a hemispherical end cap, containing the feed-through for signal and power. The cable to the lower PMT is threaded through a small hole in the ALG and runs inside the WLS tube, so that both PMTs can be supplied through one feed-through without shadowing from the cable. Fig. 5 shows a schematic drawing of the holding structure and photos of the assembled WOM prototype.

\subsection{PMT and noise}

Currently, the WOM uses the same PMT type that is also used in KM3NeT [6] (Hamamatsu R12199-02) with a passive base. In this configuration the dark noise of the PMTs has been measured to range from $(22 \pm 5) \mathrm{Hz}$ at $-10^{\circ} \mathrm{C}$ to $(31 \pm 6) \mathrm{Hz}$ at $-50^{\circ} \mathrm{C}$, more than an order of magnitude 


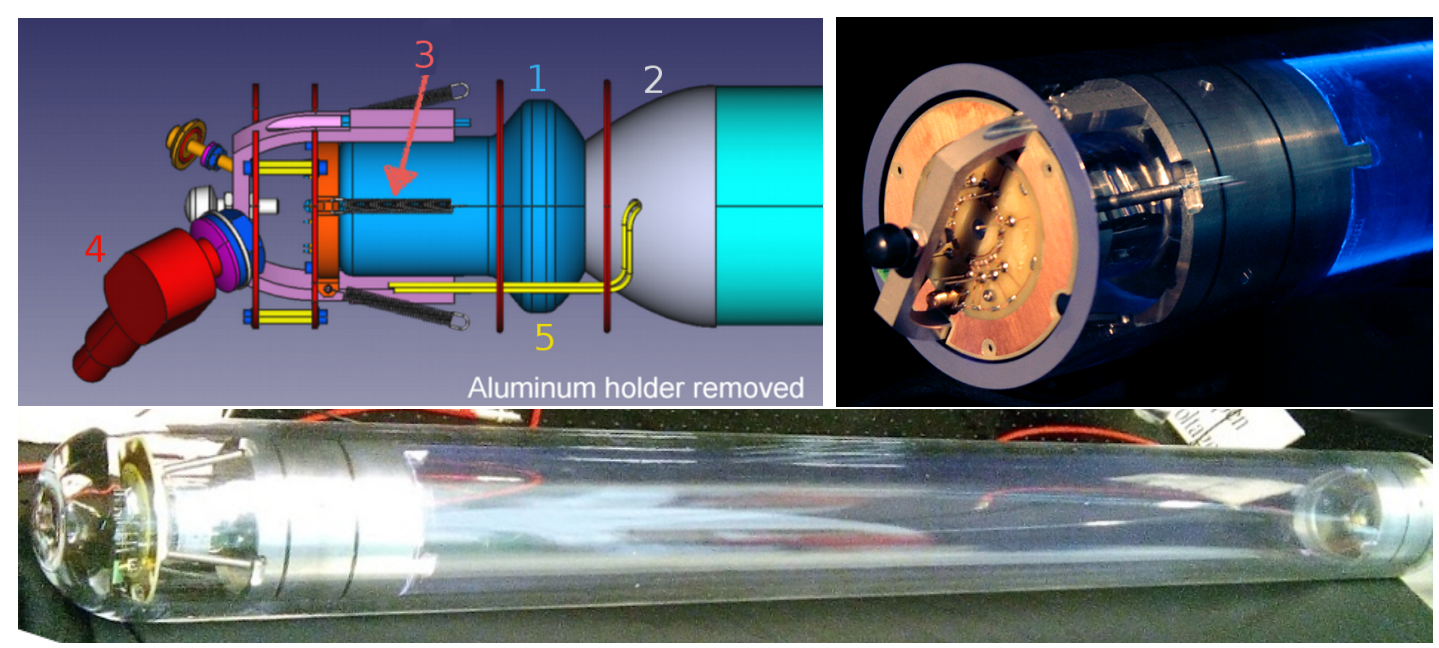

Figure 5: Upper left: A drawing of the holding structure. 1=PMT, 2=ALG, 3=springs, 4=pressure-vessel feed-through, 5=internal connecting cable (aluminum jacket not shown). Upper right: complete holding structure inside the quartz tube without end-cap. Bottom: an assembled prototype.

lower than the $\approx 500 \mathrm{~Hz}$ of the DOM. Research into improved PMTs and the radiogenic noise contributions of the quartz vessel, the WLS tube and the ALG is ongoing.

\section{WOM testing setup}

To fully characterize the WOM, we have constructed an optical setup, that will allow 2D scans of the entire tube surface. It is used to check the coating uniformity and investigate the light propagation inside the tubes. Fig. 6 shows a schematic of the setup, consisting of a Xenon lamp with a monochromator and a beam splitter using of fused silica optics for optimal UV transparency. The light beam is interrupted by a chopper wheel connected to a lock-in amplifier setup for noise suppression. A major source of PMT noise is the activation of the photo-cathode due to ambient light when exchanging a tube. This noise takes hours to subside, but with this setup it can be suppressed to a level, where measurements can be taken without any dead-time after re-mounting a tube. The photo-diode (PD) is used as reference, while a part of the light is guided to the WOM tube by the liquid light guide (LLG). Its end is mounted on an orbital wheel around the tube, so the tube can be illuminated from any angle. The orbital wheel is mounted on a linear stage, which can be driven along the entire length of the tube. At both ends the shifted light trapped in the tube is read out by calibrated PMTs. The tube is optically coupled to the PMTs using special optical gel pads, to ensure re-mounting stability. The distance between the PMTs can be altered to allow for different tube lengths with or without adiabatic light guides.

Automated scans with arbitrary step sizes in position, rotation and wavelength can be made. For each illumination spot the efficiency of the photon detection is measured, which takes about $4 \mathrm{sec}$ per spot, including sled motion. The efficiency is the convolution of the shifting efficiency $\varepsilon_{W L S}$ of the WLS (close to one), the trapping ratio $\varepsilon_{T R}$ (close to 0.75 ), the light guiding efficiency $\varepsilon_{L G}$ (unknown, depends on absorption and scattering in the tube) and the efficiency of the PMTs $\varepsilon_{P M T}$ (collection- and quantum efficiency. Known, depends on $\lambda$ ). It is defined as: 


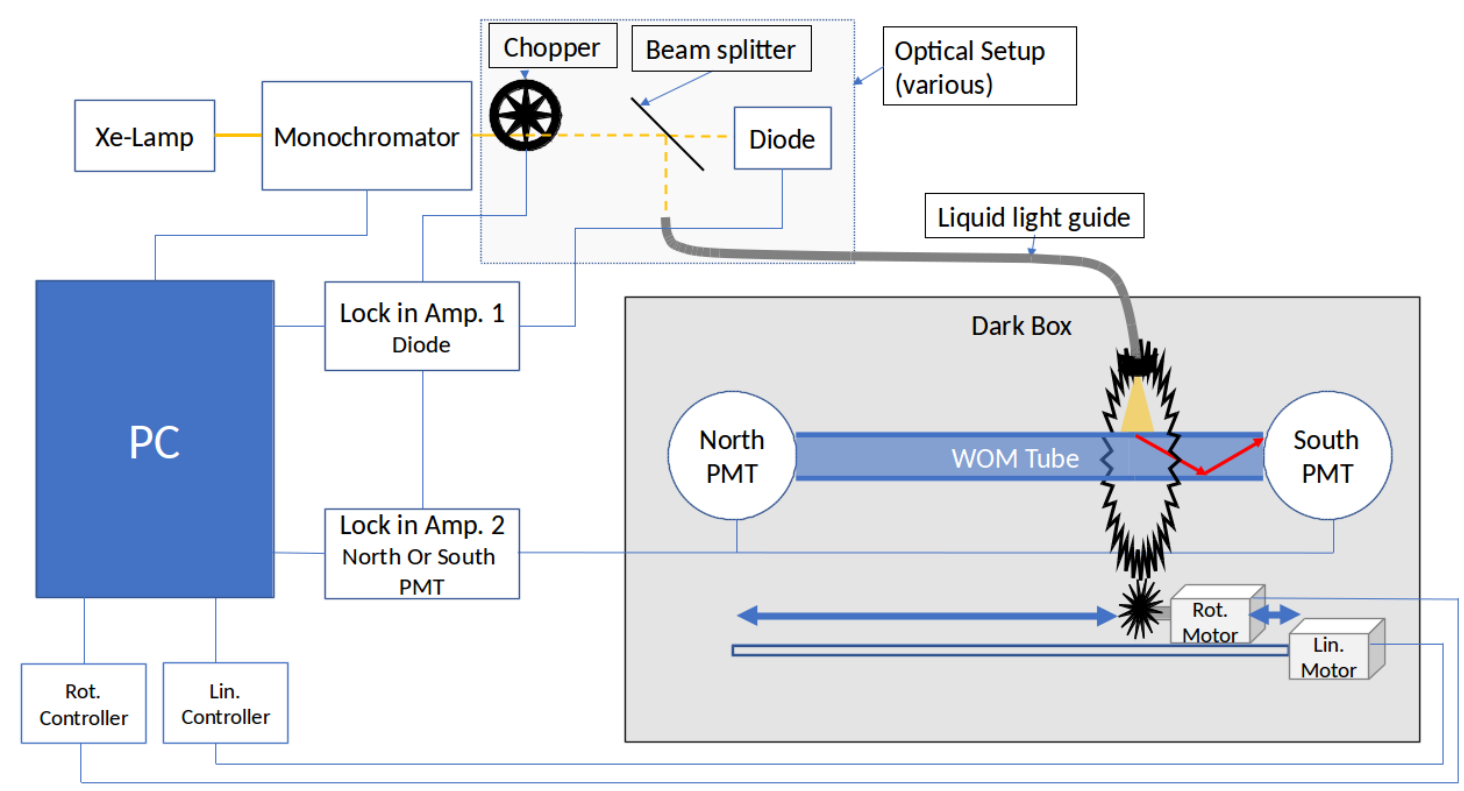

Figure 6: A schematic drawing of the tube scanner, using fused silica optics and a liquid light guide.

$$
\varepsilon_{\text {tot }}\left(\lambda_{\text {in }}\right)=\varepsilon_{W L S} \cdot \varepsilon_{T R}\left(\lambda_{\text {out }}\right) \cdot \varepsilon_{L G} \int \varepsilon_{P M T}\left(\lambda_{\text {out }}\right)=\frac{\# \text { of detected photons }}{\# \text { of injected photons }}
$$

The number of injected photons is known from the calibrated reference PD and the fixed branching ratio between the reference beam and the light intensity at the exit of the fiber. The number of detected photons is calculated from the PMT signal, it's known spectral sensitivity function and the emission spectrum of the WLS. With the photo-diode correction the system is linear over the full wavelength-range of the Xenon lamp and stable in time. Fig. 7 shows a 2D scan of the PMT signal current over illumination position. Close to the PMT the light yield is maximal. It drops off over the length of the tube due to attenuation.

\section{Simulation}

A simulation of the WOM has been integrated into the IceCube software framework, so that the in-ice performance can be simulated for different detector configurations. Muons in the energy range of $3 \mathrm{TeV}$ to $1 \mathrm{PeV}$ are injected into the ice volume. The resulting Cherenkov light emission is calculated and propagated through the ice. Photons intersecting the OM surface are stored for later processing, which uses the WOM properties. In the first step the angular acceptance, derived from Fresnel equations and Snell's law, with a refractive index of $n_{\text {ice }}=1.33, n_{\text {glass }}=1.50$ and $n_{\text {air }}=1.00$, is applied, followed by the measured wavelength acceptance of the WLS tube. This includes the efficiency of the WLS and the propagation efficiency which together give about $50 \%$ between $\lambda=250-400 \mathrm{~nm}$. This is combined with the PMT acceptance convoluted with the emission spectrum. Since the probability of losing a photon that propagates in the tube is lower when it hits close to one of the PMT, a position dependent correction function was introduced based on previous measurements. In a first simulation we look at the total number of photons detected as 


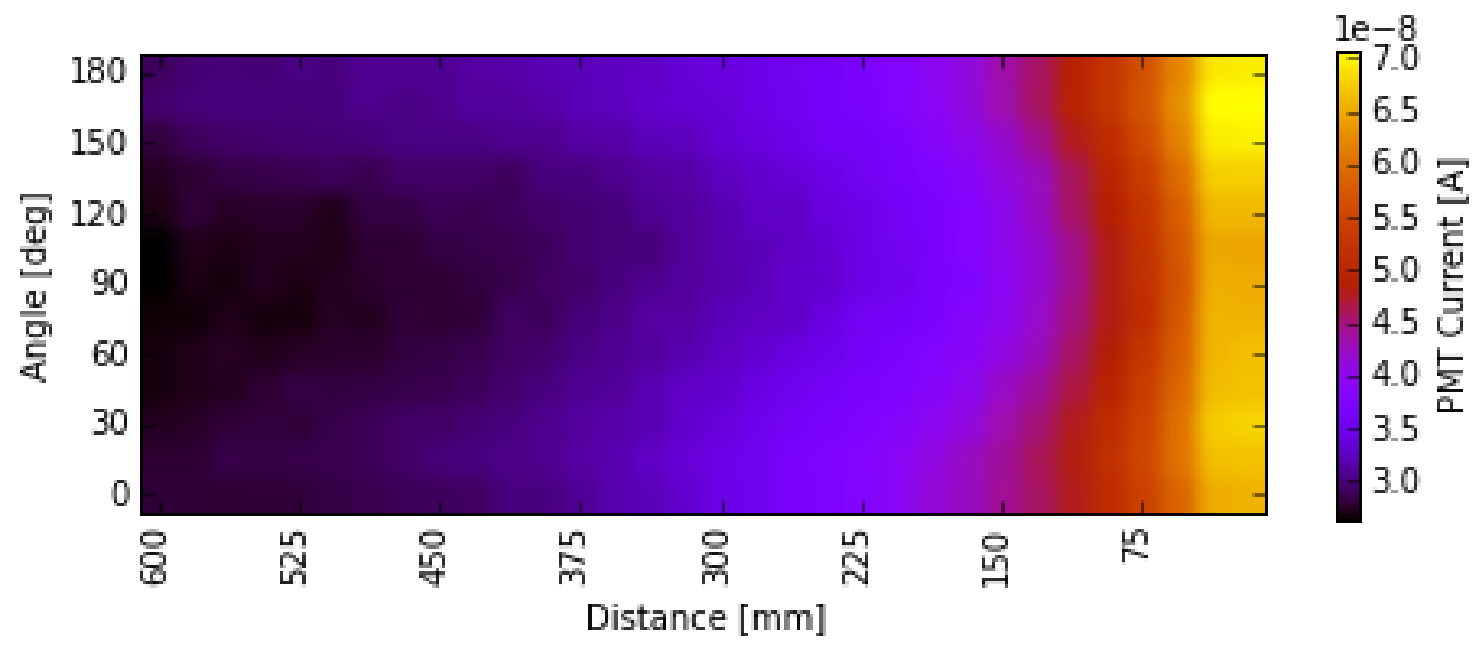

Figure 7: 2D scan of the local efficiency of the WLS tube. Shown is the PMT signal current over longitudinal position in $\mathrm{mm}$ and rotational position in degree for an incident wavelength of $\lambda=375 \mathrm{~nm}$. The PMT is located to the right. The variation over a rotation at fixed distance is $\leq 10 \%$

a function of energy and distance from the muon track. We simulate $2 \cdot 10^{5}$ events with an energy power-law index of -1.4 in the so called sunflower 240 detector geometry ${ }^{2}$ [2]. For comparison the pDOM, an upgraded version of the IceCube-DOM is used with an identical geometry and event set. Fig. 8 shows the number of photons detected per muon event, plotted against the event's energy. Over the whole range the WOM detects a factor of $1.7 \pm 0.1$ more photons than the pDOM.
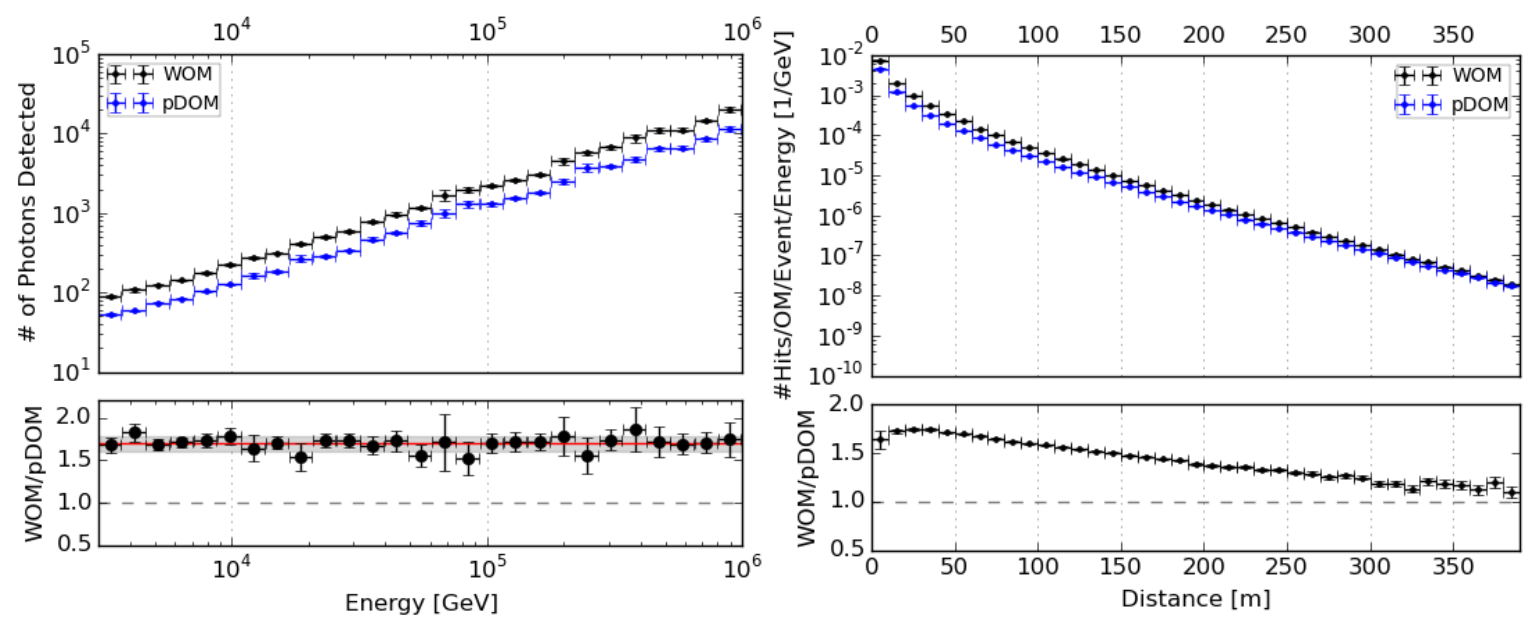

Figure 8: Comparison of the number of photons detected as a function of energy for the WOM and the pDOM in the sunflower 240 geometry. It can be seen, that the WOM detects a factor of $1.7 \pm 0.1$ more photons than the pDOM for all energies.

Figure 9: Comparison of the number of photons per energy detected as a function of the closest distance to the muon track for the WOM and the pDOM in the sunflower 240 geometry. The WOM detects more photons than the pDOM for all relevant distances.

\footnotetext{
${ }^{2}$ Other geometries perform similar for the parameters shown.
} 
In Fig. 9 the distance between muon track and the individual OMs is investigated. The photon number is plotted per energy, per event and OM against this distance. The WOM performance decreases as a function of distance due to shorter attenuation-length for the UV photons in the ice, but stays better than that of the pDOM for all relevant distances. The plateau at very short distances (i.e. for unscattered photons) is caused by the different angular acceptance of the modules.

Detailed measurements of the WOM time response are ongoing and will be implemented in the simulation for a realistic arrival time modeling. Additionally the use of a super-bialkali version of the PMT might increase the efficiency by up to $45 \%$. Any efficiency values for new WLS paints that are studied can also be directly included in the simulation framework.

\section{Summary and Outlook}

The WOM has proven to be a very promising low noise, high efficiency and low cost optical module, but further improvements of several components and simulations are ongoing. A reliable mounting structure has been developed and a prototype has been assembled. The WLS developed for the WOM shifts light from the $\lambda \approx 250-400 \mathrm{~nm}$ to $\lambda>400 \mathrm{~nm}$. Measurements of the light capture and transport efficiency showed values up to 50\%[8]. However, the efficiency strongly depends on coating quality and uniformity. Further studies into the optimization of the WLS coating are ongoing and a dedicated semi-automated measuring setup has been built to quantify any future coatings. The adiabatic light guides developed for the WOM have been successfully coupled to the WOM tube with an UV-curing glue, but further optimization of the gluing process are ongoing to minimize the light loss. The quartz vessel has been pressure tested and has been proven to be vacuum tight for more than a year. In simulations the WOM has been shown to detect more photons than the pDOM for all energies, types of events and detector geometries for IceCube-Gen2. Beyond IceCube-Gen2, it's high efficiency and low noise make this optical module the ideal candidate for the conceptualized MICA supernova detector [9]. The performance of the WOM can be improved even further by using new PMTs with a higher quantum efficiency or newly developed WLS. The WOM was developed for the IceCube-Gen2 experiment. However, it can be conceptually adapted to other experiments that profit from it's UV detection capabilities, such as SHiP. [7]

\section{References}

[1] IceCube Collaboration, M.G. Aartsen et al., JINST 12 (2017) P03012;

[2] IceCube-Gen2 Collaboration, arXiv:1412.5106v2 [astro-ph.HE]

[3] IceCube-Gen2 Collaboration, J. Phys. G 44 (2017) 054006

[4] IceCube PINGU Collaboration, P. Sandstrom et al., AIP Conf. Proc. 1630 (2014) 180-183.

[5] IceCube Collaboration, PoS ( ICRC2015) 1134, 2016

[6] KM3NeT Collaboration, Letter of Intent for KM3NeT 2.0, arXiv:1601.07459v2 [astro-ph.IM]

[7] SHiP Collaboration, arXiv:1504.04956, April 2015

[8] IceCube-Gen2 Collaboration, D. Hebecker et al. EPJ Web of Conferences 116, 01006 (2016)

[9] Sebastian Böser et al., Astropart. Phys. 62, March 2015, Pages 54-65 